\title{
Associations évoquées par le changement climatique chez des citoyens français et norvégiens
}

\author{
Kjersti Fløttum et Øyvind Gjerstad (Dép. des Langues étrangères - \\ Université de Bergen, Norvège) \\ Endre Tvinnereim (NORCE Norwegian Research Centre / \\ Dép. d'administration et de théorie organisationnelle - Université de \\ Bergen, Norvège)
}

\begin{abstract}
Communication related to climate change as well as to its consequences constitutes a major challenge; all the more so since climate is a nonobservable phenomenon, in stark contrast to the weather. In this perspective, language plays a crucial role in the conceptualisation and the framing of climate change discourse.

In this paper, French and Norwegian data stemming from representative surveys in the two countries are compared. The participants are asked to answer an openended question concerning their conceptions of the expression "climate change". The French data are collected from a survey undertaken in 2016 by ELIPSS at the Sciences Po (Institut d'études politiques de Paris). The Norwegian data are generated from a survey undertaken in 2013 by the Norwegian Citizen Panel/DIGSSCORE, at the University of Bergen. The answers are first analysed through a semi-automated structural topic modeling (STM) and then assessed through an in-depth manual evaluation. Further linguistic and enunciative analyses are undertaken of a selection of the respondents' answer provided by the surveys.

Given the difference in the energy mix of the two countries, different associations are expected to appear from the French and the Norwegian participants. At the same time, with the common global dimension of climate change, it seems reasonable to expect some similar associations concerning the reality of the changes, the consequences and the measures of adaptation or mitigation proposed or undertaken by the two countries. These issues are discussed within the theoretical frame of enunciation, including perspectives related to concession in a polyphonic perspective and to deontic modality.
\end{abstract}

Keywords: climate change discourse, open-ended survey questions, French and Norwegian discourse, structural topic modelling, concession, deontic modality

\section{Introduction}

Le climat en tant que tel n'est pas un phénomène qui s'observe, et cela en grand contraste avec le temps comme la pluie, la chaleur, la neige, et aussi en contraste avec les 
conséquences du changement climatique. Voilà une des raisons de la problématique concernant la communication portant sur ce défi global, avec des conséquences se manifestant dans la plupart des cas au niveau local. En outre, le changement climatique est en effet passé d'un phénomène principalement géophysique à un phénomène social, politique, économique, éthique, culturel et communicationnel (Hulme 2013), où le rôle du langage devient primordial (Dahl \& Fløttum 2014 ; Fløttum 2016, 2017 ; Fløttum \& Dahl 2014 ; Fløttum \& Gjerstad 2016 ; Fløttum et al. 2014 ; Moser 2016 ; Nerlich et al. 2010 ; Tvinnereim et al. 2017). Le mélange de perspectives concernant les effets géophysiques avec la politique se manifeste clairement dans la citation suivante, de la première ministre de la Norvège, Erna Solberg (du 20 janvier 2015) : « Nous n'avons pas fait bouger la banquise. Elle a bougé elle-même. » ${ }^{1} \mathrm{La}$ première ministre a fait ces remarques suite à des critiques contre la décision de la part du gouvernement de retracer la ligne qui sépare la mer de la banquise. Elle voulait ainsi contrer l'impression qu'il s'agissait d'une mesure politique pour ouvrir la zone à l'exploration pétrolière, en soulignant que le processus était naturel et donc non pas influencé par les possibilités économiques de l'Arctique.

Le langage utilisé joue un rôle essentiel dans la construction et le cadrage du discours portant sur le réchauffement de notre planète. Ce défi communicationnel a été le point de départ d'un projet monté à l'Université de Bergen, le projet LINGCLIM, abréviation de Représentations LINGuistiques du discours portant sur le changement CLIMatique et leurs interprétations individuelles et collectives, constituant le cadre du présent article. Notre question de recherche principale a porté sur le rôle du langage dans le débat portant sur le climat, et cela dans une collaboration interdisciplinaire, où nous, les linguistes et politologues, ont collaboré avec des chercheurs de climat, média, psychologie et informatique. Notre objectif est de développer une contribution innovatrice et nécessaire, en théorie et en empirie, à la base de connaissances sur laquelle se fondent les décisions et actions politiques et sociétales portant sur le changement climatique. Plus spécifiquement, il s'agit de révéler la relation entre les représentations linguistiques et les interprétations qu'en font différents publics ainsi que d'explorer l'impact des encadrements ('frames') conflictuels, les attitudes, les émotions et les perspectives sur l'avenir.

Les composantes principales de la recherche consistent dans des analyses linguistiques et discursives de différents genres textuels, des analyses (semi-)automatiques de larges corpus textuels, différentes méthodes computationnelles, ainsi que des enquêtes et expériences. Les matériaux textuels sur lesquels nous avons travaillé sont hétérogènes: il s'agit de discours scientifique (documents GIEC/IPCC), discours politique (livres blancs, programmes politiques), documents de l'ONU, discours des médias traditionnels et des réseaux sociaux, et enfin ce que nous appelons « discours d'enquête » (« survey discourse » en anglais; Fløttum 2016) - à savoir les réponses à des questions ouvertes, librement formulées par les répondants d'une enquête (voir section 2). L'objectif du présent article est de discuter les résultats de deux enquêtes, entreprises au sein de notre groupe, se basant sur une question ouverte, en Norvège en 2013 et en France en 2016. Nous pensons que les données que nous avons obtenues fournissent de nouvelles connaissances sur les éventuelles possibilités et contraintes que présentent les interprétations des citoyens du défi climatique - des interprétations que doivent connaître les décideurs ayant à adopter différentes mesures pour atténuer les conséquences du changement climatique.

\footnotetext{
${ }^{1}$ Citation originale : «Vi har ikke flyttet iskanten. Den har flyttet seg selv.», du 20 janvier 2015.
} 
Concernant le choix d'étudier les résultats d'enquêtes entreprises en France et en Norvège, nous pensons qu'une telle comparaison pourra nous donner de nouvelles connaissances sur la problématique en question. Notre point de départ a été la différence notable en ce qui concerne leurs bouquets énergétiques. La Norvège est dans une situation où l'électricité est presque uniquement produite par des barrages hydroélectriques ; en même temps, elle est un grand exportateur de pétrole et de gaz, qui en plus voudrait bien être au front dans les questions d'environnement et de climat. Voilà ce qui est souvent considéré comme le paradoxe norvégien. En comparaison, en France, près des $3 / 4$ de la production d'électricité est générée par le parc nucléaire; en même temps on y a adopté une loi de transition énergétique pour la croissance verte qui prévoit de réduire cette proportion à $50 \%$ d'ici 2025. Il semble donc intéressant de voir dans quelle mesure leur conception du défi climat pourra être similaire ou différent (pour une présentation plus détaillée des différences entre les deux pays, voir Steentjes et al. 2017).

Après une brève description de nos données et des approches d'analyse utilisées (2), nous présenterons les résultats d'une analyse semi-automatique des thèmes associés avec 'changement climatique' dans des réponses françaises et norvégiennes (3.1). Ensuite nous entreprendrons une analyse plus linguistique concernant les représentations des expressions d'obligation et de responsabilité (3.2). La dernière partie des analyses portera sur le rôle de structures concessives (3.3). Notre article se terminera par de brèves remarques finales (4).

\section{Matériaux et méthodes}

Nous ferons ici une description des données provenant de deux enquêtes, entreprises en France et en Norvège, incluant une question ouverte, identique dans les deux pays, afin de savoir plus de leurs associations évoquées par le phénomène du changement climatique.

La raison pour laquelle nous avons choisi de travailler sur de tels matériaux, que nous obtenons par des enquêtes d'opinion, réside dans le fait que les questions ouvertes nous fournissent des données beaucoup plus riches que les réponses des questions fermées. Il est vrai que les questions fermées avec des alternatives de réponse étant pré-établies sont les plus utilisées dans les questionnaires. Une raison en est qu'elles facilitent le traitement des résultats d'une enquête. Mais une question fermée, c'est une simplification réductrice (Stoneman et al. 2013). En contraste, les questions ouvertes permettent de recueillir des avis de façon détaillée. Elles peuvent stimuler l'intérêt du répondant pour le questionnaire en lui donnant le sentiment qu'on s'intéresse à lui et qu'il peut s'exprimer librement. Les réponses obtenues sont variées - longues ou courtes - et nécessitent donc un travail de traitement relativement vaste (on n'entrera pas dans les détails de ce travail ; pour en savoir plus, voir Tvinnereim et al. 2017).

La question dans les deux enquêtes a été formulée comme suit (le norvégien est traduit en français) :

«A quoi pensez-vous quand vous entendez ou lisez l'expression "changement climatique"? Merci de noter la première idée qui vous vient à l'esprit. Toutes les réponses nous intéressent : cela peut être un mot, plusieurs mots ou plusieurs phrases. »

Il s'agit donc d'une question qui a été posée dans deux enquêtes, représentatives, et assurées par des institutions scientifiques, à savoir l'Université de Bergen et les SciencesPo, Paris : 
1) Enquête de niveau national, par internet, 2013 : Norwegian Citizen

Panel/DIGSSCORE -

Panel de Citoyens Norvégiens ; ci-après PCN

2155 réponses; total de 21470 mots; variations des réponses entre 1 et 310 mots

2) Enquête de niveau national, par internet, 2016 : ELIPSS $^{2}$, assurée par Sciences Po, en collaboration avec le CNRS; ci-après ELIPSS

2687 réponses; total de 15119 mots; variations des réponses entre 1 et 49 mots.

Il y a une différence en ce qui concerne l'année où les enquêtes ont été entreprises 2013 pour PCN en Norvège et 2016 pour ELIPSS en France. Ce paramètre pourrait être important dans l'interprétation des résultats ; on y reviendra.

A part cette différence, on observe que le nombre de réponses est plus élevé pour ELIPSS que pour PCN, tandis que le nombre de mots est plus élevé en Norvège qu'en France. Cela signifie que la longueur moyenne des réponses est différente - pour PCN : 10 mots, et pour ELIPSS : 5,6 mots. En effet, il y a plus de réponses longues (plus de 10 mots) dans les données norvégiennes, ce qui pourra indiquer un engagement dans la problématique un tant soit peu plus fort parmi les répondants.

Le résultat de cette enquête constitue une grande quantité de données hétérogènes en longueur, en thème et dans une certaine mesure en style (voir les exemples en 3.1-3.3). Cette hétérogénéité a nécessité un traitement complexe, quantitatif aussi bien que qualitatif. Pour savoir comment les réponses se répartissent sur des thèmes, on a utilisé l'outil «Structural Topic Modelling» (Roberts et al 2014). Cet outil semi-automatique propose une division en thème basée sur la fréquence de mots ainsi que sur l'exclusivité, c'est-à-dire les mots qui distinguent un sous-corpus d'un autre. Pour plus de détail sur cette méthode, on renvoie à l'article de Tvinnereim \& Fløttum 2015.

\section{Analyses}

\subsection{Comparaison thématique entre associations françaises et norvégiennes}

Les résultats de l'analyse thématique nous donnent une première impression des associations évoquées par la collocation 'changement climatique'. Pour la Norvège, les associations concernent avant tout les thèmes suivants : temps extrême/des changement de température/la fonte des glaces. Certains des répondants sont plus préoccupés par l'avenir et les conséquences pour les futures générations; ensuite il y en a qui réfutent la question et discutent de l'attribution ou des causes du changement, référant surtout aux cycles naturels du climat, et enfin, il y a des répondants qui accusent les êtres humains d'une consommation exagérée et par là d'être responsables d'activités destructives pour l'environnement.

Pour ce qui est des répondants français, on voit le même type d'associations dans leurs réponses incluant le temps/la température/le réchauffement et la montée d'eau. Cependant, les Français sont surtout inquiets de ce qu'ils appellent catastrophe naturelle, et en particulier ils

\footnotetext{
${ }^{2}$ ELIPSS (L'étude longitudinale par internet pour les sciences sociales): Un panel de 3500 personnes équipées de tablettes tactiles connectées à internet pour répondre régulièrement à des études conçues par des chercheurs en sciences humaines et sociales sur des sujets d'intérêt général.
} 
ont observé des saisons perturbées. En plus, comme les répondants norvégiens, ils accusent les êtres humains d'activité destructive pour le climat.

Sans entrer plus dans les détails, il faut reconnaître la différence qu'il peut y avoir concernant les conceptions de 2013 en Norvège et celles de 2016 en France. Pendant cette période de trois années a eu lieu, par exemple, la conférence internationale de COP21 à Paris ainsi que de nouveaux rapports publiés par le GIEC, le Groupe d'experts intergouvernemental sur l'évolution du climat. Cela étant, dans la mesure où on peut en tirer des différences notables, il semble que les Français soient plus préoccupés par les perturbations des catastrophes naturelles, et que les Norvégiens portent plus d'attention aux conséquences pour la société et les humains. Une particularité des Français est leur conception de saisons perturbées, ou la dite disparition des saisons.

Cette analyse thématique, entreprise dans une collaboration fructueuse entre linguistes et politologues, nous fournit un contexte pertinent pour aborder des analyses plus linguistiques des données en question. De cette manière, les approches quantitatives combinées avec des approches qualitatives contribueront à des interprétations plus approfondies de nos données.

\subsection{Analyses des conceptions d'obligation et de responsabilité}

En nous intéressant à la manière dont les répondants s'expriment, nous pensons qu'une approche dans une perspective énonciative contribuera à une interprétation intéressante de la recherche portant sur les enquêtes telles que celles qu'on présente ici. Une telle approche qualitative nous semble nécessaire pour savoir ce que sont les associations des répondants. Il s'agit d'une série de points essentiels concernant les relations entre acteurs et arguments, les attitudes liées à différents problèmes, et la polyphonie ou caractère dialogique souvent caractérisant les réponses longues. Pour notre étude comparative, cette approche nous aidera mieux à révéler des différences éventuelles entre les Français et les Norvégiens. Nous allons ici nous limiter à quelques exemples illustrant comment les répondants s'expriment par rapport à des questions d'obligation et de responsabilité, concernant dans beaucoup de cas la modalité déontique.

A) Exemples traduits du norvégien

i. Det er krevende men helt nфdvendig å fase ut olje, kull og gass.

Il est difficile mais absolument nécessaire de supprimer progressivement le pétrole, le charbon et le gaz.

ii. Alvorlig. Noe må gjøres.

C'est grave. Il faut faire quelque chose.

iii. Vi må bry oss, selv om vi er skånet for det meste i Norge.

Nous devons nous sentir concernés, bien qu'on soit à l'abri du pire en Norvège.

$i v$. Norge bør gå foran som et godt eksempel. La Norvège devrait montrer l'exemple.

v. Hvorfor skal lille Norge ligge på toppen for å bekjempe dette???

Pourquoi la petite Norvège devrait-elle s'imposer la responsabilité de lutter contre cela ??? 
Les exemples 1-2 expriment la nécessité de faire quelque chose, mais dans des constructions impersonnelles, sans acteurs. Dans l'exemple 3, des acteurs sont inclus, représentés par le pronom «nous », mais comme nous le savons, ce pronom est vague, indéfini, et nous permet d'en faire différentes interprétations. Dans les exemples 4 et 5 , c'est le pays - la Norvège - qui est mise en cause, mais de manière tout à fait différente dans les deux réponses : en 4 , on impose à la Norvège de se montrer comme un pays qui assume ses responsabilités, tandis qu'en 5, dans la forme d'une question, une opinion implicite indique que la Norvège est trop petite pour en pouvoir faire quelque chose - une excuse pas très bien fondée.

B) Exemples français

i. Il faut agir.

ii. Bouleversements de la planète il faut que les hommes réagissent.

iii. Avant de nous faire chier avec [ç]a les grandes usines devraient se poser des grandes questions !!

iv. Il faut absolument s'entendre entre les pays pour stopper l'échauffement de la terre.

v. Dramatique car certains dirigeants promettent de tout faire pour l'environnement et finalement continuent à polluer alors que ce sont toujours les mêmes tels la France, conscients du problème, qui essaient d'y remédier.

Nous observons plusieurs similarités avec les réponses norvégiennes. Tous les exemples expriment la nécessité de faire quelque chose, sans acteur en une construction impersonnelle comme en 1, ou avec des acteurs inclus plus ou moins déterminés : «les hommes » en 2, «les grandes usines » en 3, «les pays » en 4. L'exemple 5 n'est pas tout à fait clair, mais le répondant semble exprimer une responsabilité entreprise par la France.

Dans les deux enquêtes on trouve aussi différentes réponses qui questionnent ou tout simplement réfutent la problématique entière :

C) Mise en question du problème

i. On doit être vigilant sur les informations qu'on reçoit.

ii. Bah !! Pas à grand-chose. [...] La terre ne s'est pas arrêté de tourner !! Alors pour moi le blabla !!

iii. Quels changements de vie cela va-t-il avoir pour nous? Quelles conséquences pour la planète ? Que peut-on y faire

iv. Det er trolig menneskeskapt, men ikke helt sikkert. C'est probablement anthropique, mais pas tout à fait certain.

v. Jeg tenker ingenting for jeg bryr meg ikke om klimakrisen som er en stor svindel. Je ne pense rien, parce que je m'en fiche de la crise climatique qui est une grande escroquerie.

En conclusion des réponses montrées ici : Il y a une grande variation, on voit aussi bien la présence que l'absence d'engagement. La responsabilité ou l'obligation de faire 
quelque chose est bien là, mais pour ce qui est des acteurs dans les actions éventuelles à entreprendre, c'est moins clair - et cela dans les deux pays. Enfin, dans les deux pays il y a des répondants qui ne s'en préoccupent pas ou qui simplement réfutent toute la problématique.

D'un point de vue quantitatif, en considérant le nombre d'occurrences d'une sélection de différentes expressions, nous observons des contrastes intéressants entre les deux enquêtes :

\begin{tabular}{|lclc|}
\hline France-ELIPSS & Fréquence relative en \% & Norvège-PCN & Fréquence relative en \% \\
\hline devoir (au présent) (1), & 0,01 & bør (30), & 0,14 \\
devr* (2) & 0,01 & burde (17) & 0,08 \\
\hline il faut (14), & 0,09 & må (140), & 0,65 \\
il faudr* (1) & 0,01 & måtte (2) & 0,01 \\
(ne) pas (92) & 0,61 & ikke (241) & 1,12 \\
mais (22) & 0,15 & men (138) & 0,64
\end{tabular}

Tableau I : Répartition de quelques éléments linguistiques.

Parmi les chiffres de la Table 1, il y a surtout une différence qui peut surprendre, concernant les expressions qui peuvent servir à exprimer une modalité déontique, une obligation, notamment les diverses formes de «il faut» en français et du verbe «må » en norvégien. Ces expressions sont beaucoup plus nombreuses dans les réponses des Norvégiens que dans celles des Français. Une différence frappante entre les deux pays est donc l'absence presque totale d'expressions déontiques parmi les répondants français.

Quelle pourra en être l'explication? Pour l'instant nous nous contentons de dire que ces différences n'indiquent pas vraiment une présence plus importante d'agents « responsables » dans l'enquête norvégienne. Car en regardant les différents agents associés aux expressions déontiques, plus de 70 pour cent sont indéterminés, à travers des constructions impersonnelles, ou bien par l'emploi de pronoms comme nous (vi), on (man) ou tout le monde (alle).

Autrement dit, si quelques répondants norvégiens pointent le doigt sur les pays riches, les citoyens norvégiens, ou bien le gouvernement, la majorité des occurrences se contentent d'attribuer la responsabilité d'agir à un nous très flou ou bien à personne. Peut-être ce manque de responsabilité est le reflet de la complexité de la question climatique : si tout le monde est plus ou moins coupable, comment imposer la responsabilité d'agir à qui que ce soit?

Nous observons aussi une fréquence différente, bien que moins éclatante, en ce qui concerne l'emploi de la négation (ne-pas et ikke). Dans la mesure où il s'agit de négations polémiques, cette différence pourra indiquer une polyphonie plus marquée dans les réponses norvégiennes, où plusieurs points de vue en opposition les uns aux autres sont introduits dans les réponses (pour une étude des négations dans le discours portant sur le climat, voir Gjerstad \& Fløttum 2017). Il y a également des différences considérables pour ce qui est de l'emploi du connecteur concessif mais, qui apparaît avec une fréquence relative plus élevée dans les réponses norvégiennes que celles des Français. Cette différence indique que les Norvégiens discutent du changement climatique de manière plus délibérative que les Français. Cette 
impression est renforcée par la longueur moyenne des réponses des deux sondages. Nous considérerons le fonctionnement des structures concessives d'une manière plus approfondie dans la section suivante.

\subsection{Le rôle de structures concessives}

La particularité des structures concessives en mais, dans une perspective polyphonique (Nølke et al. 2014), est de nuancer l'argumentation, en intégrant deux points de vue qui ont des orientations argumentatives contraires. Or, afin de juger de l'effet discursif de ce marqueur linguistique, il faut regarder de plus près les occurrences que l'on en trouve dans les deux corpus. D'abord, il faut faire la distinction entre mais adversatif et mais concessif (Birkelund 2009). Dans cette étude, nous nous intéressons surtout à ce dernier, qui sert à mettre en relation deux arguments qui s'opposent de manière indirecte, en s'orientant vers des conclusions opposées. La catégorie peut s'illustrer à travers l'exemple suivant, tiré d'une réponse française (c'est nous qui soulignons dans les exemples ci-dessous) :

(1) Le changement climatique 'rechauffement' est vrai mais sera inversé dans une trentaine d'années par un refroidissement, globalement à l'échelle de la vie de la terre, on est plutôt en refroidissement.

Le répondant concède l'existence du réchauffement, argument qui s'oriente vers la conclusion implicite qu'il faut réduire les émissions. Cependant, il présente ensuite un contreargument, que ce réchauffement sera suivi d'un refroidissement, renversement qui sert d'appui à la conclusion que l'action n'est pas nécessaire.

Contrairement à ce mais concessif, le mais adversatif n'oppose pas deux arguments de manière indirecte, mais se combine avec la négation pour « relier deux segments discursifs dont le dernier rectifie le premier» (Birkelund 2009). Cet emploi de mais apparaît dans l'exemple suivant :

(2) Pollution de la part des entreprises. Fin de la terre si ça continue ainsi et ce n'est pas à cause de nous simple citoyen mais les usines, les lobbys y sont en partie responsable de ce changement climatique.

Ici, il n'y a pas d'orientation vers des conclusions implicites et contraires, mais la rectification du premier contenu, que le locuteur juge faux, par le deuxième.

Dans les corpus norvégien et français, les deux usages de mais se répartissent comme suit :

\begin{tabular}{|c|c|c|c|}
\hline \multicolumn{2}{|l|}{ Norvégien } & \multicolumn{2}{|l|}{ Français } \\
\hline Concessif & Adversatif & Concessif & Adversatif \\
\hline 131 & 7 & 20 & 2 \\
\hline
\end{tabular}

Tableau II : Répartition du connecteur mais.

Nous avons constaté que la concession s'emploie pour mettre en relation des points de vue différents, des arguments et contre-arguments, afin de nuancer la position argumentative du locuteur. L'emploi de mais concessif au sujet du changement climatique indique donc un besoin chez les répondants de prendre en compte la complexité de cette thématique. A y 
regarder de plus près, quels sont les sujets qui méritent une telle stratégie discursive, aux yeux des répondants norvégiens et français ?

En effet, dans le corpus norvégien, il y a un nombre limité de thèmes récurrents, entre autres la responsabilité d'agir, et les priorités de mesures possibles, comme dans les deux exemples suivants :

(3) Norge skal gå foran som et godt eksempel på klimatiltak, men vi kan ikke redde verden alene.

La Norvège doit être exemplaire dans ses mesures climatiques, mais nous ne pouvons pas sauver le monde tous seuls.

Ici, la question de responsabilité se discute dans un cadre international, opposant la Norvège au reste du monde. ${ }^{3}$

(4) Jeg liker at vi kjemper for naturen og miljøet, jeg tror det er viktigst for helse og frisk natur og turisme. men skal vi redusere klimagasser må vi heller prioritere en mye bedre infrastruktur, for å få transport raskere og sikrere fra A til B, enn å fokusere på å bekjempe dette med skatter og avgifter.

J'aime bien que nous luttons pour la nature et l'environnement, je pense que c'est important surtout pour la santé, la nature saine, le tourisme, mais si nous devons réduire les gaz à effet de serre, il faut plutôt donner la priorité à une infrastructure améliorée, afin d'assurer un transport plus rapide et sûr entre A et B, que de vouloir combattre le problème avec des impôts et des taxes.

Dans cet exemple, ce sont les priorités entre les mesures possibles qui se discutent. Ce thème n'est pourtant pas présent dans le premier argument de la structure concessive, seulement dans le deuxième. Or, en tenant en compte que l'argument suivant men doit être considéré comme le plus important, le thème qu'il représente est par conséquent le thème dominant de la structure.

Après une lecture de tous les 131 exemples de men concessif, nous sommes arrivés aux catégories thématiques suivantes :

\begin{tabular}{|ccccccc|}
$\begin{array}{c}\text { Attribution } \\
\text { de causes }\end{array}$ & $\begin{array}{l}\text { Responsabilité } \\
\text { d'agir }\end{array}$ & $\begin{array}{l}\text { Priorités de } \\
\text { mesures }\end{array}$ & $\begin{array}{c}\text { Gravité de } \\
\text { la situation }\end{array}$ & $\begin{array}{l}\text { Perturbations } \\
\text { et destruction }\end{array}$ & Autres & Total \\
\hline 57 & 18 & 15 & 11 & 11 & 19 & 131 \\
\hline $43,5 \%$ & $13,7 \%$ & $11,5 \%$ & $8,4 \%$ & $8,4 \%$ & $14,5 \%$ & $100 \%$ \\
\hline
\end{tabular}

Tableau III : Répartition des thèmes observés dans des constructions avec men (norvégien).

On constate que près de la moitié des structures concessives concernent la question de l'attribution du phénomène des changements climatiques. Autrement dit, les répondants norvégiens se servent de men pour discuter de la cause du changement climatique, souvent en affirmant la coexistence des causes naturelles et humaines, comme dans les exemples suivants :

\footnotetext{
${ }^{3}$ La question de la responsabilité de la Norvège vis-à-vis des grandes puissances a été identifiée comme un thème important des réponses données aux questions ouvertes du PCN (voir Tvinnereim et al. 2017).
} 
(5) Noe kan vare menneskeskapt men det er umulig å vite hvor mye fordi klimaet har $i$ historisk perspektiv gjennomgått veldig store endringer. Klimaendringer fant sted for det bodde mennesker på jorden.

En partie ce peut être anthropique, mais il est impossible de savoir par combien parce que le climat a subi des changements considérables, dans une perspective historique. Les changements climatiques ont eu lieu avant l'arrivée des êtres humains dans le monde.

(6) Klima har allid forandret seg gjenom tidene. Men nå vet jeg at vi mennesker forandrer klimaet til det verre pga forurensing.

Le climat a toujours changé de tout temps. Mais maintenant je sais que nous les êtres humains changent le climat pour le pire à cause de la pollution.

Ces deux exemples combinent les mêmes arguments dans la structure concessive, mais de manière opposée. En effet, l'ordre détermine l'orientation argumentative de la structure, étant donné que l'argument final se présente comme le plus pertinent. Ainsi, l'exemple (5) s'oriente vers l'inaction, car l'existence de changements climatiques naturels est affirmée en fin de construction. Par contre, (6) s'oriente vers l'action, du fait que la structure se termine par le côté anthropique des changements climatiques. Parmi les 57 structures concessives appartenant à la catégorie «attribution», nous avons identifié 16 cas qui combinent les arguments de la même manière que l'exemple (5), et 24 cas qui ressemblent à l'exemple (6). Les répondants norvégiens font donc preuve d'un besoin de prendre en compte ce qu'ils considèrent comme la complexité des changements climatiques, une complexité dont ils sont capables de rendre compte grâce à la possibilité de l'expression libre dans la réponse à la question ouverte.

Qu'en est-il des répondants français ? Parmi les 20 structures concessives en mais, combien de catégories thématiques peut-on identifier ? En effet, on voit en partie les mêmes catégories que dans le corpus norvégien :

\begin{tabular}{|llllll} 
Attribution & Priorités & $\begin{array}{l}\text { Manque } \\
\text { d'action }\end{array}$ & $\begin{array}{l}\text { Perturbations } \\
\text { et destruction }\end{array}$ & Indéterminé & Total \\
\hline $\mathbf{5}$ & 5 & 1 & 6 & 3 & 20 \\
\hline $\mathbf{2 5} \%$ & $25 \%$ & $5 \%$ & $30 \%$ & $15 \%$ & $100 \%$ \\
\hline
\end{tabular}

Tableau IV : Répartition des thèmes observés dans des constructions avec mais (français).

La rareté de structures concessives dans le corpus français nous empêche de faire une comparaison globale avec celles du corpus norvégien. On peut néanmoins noter certaines ressemblances dans la catégorie « attribution»:

(7) Montées des eaux des océans, changement de climat mais est-ce que c'est dû essentiellement à la pollution ou à autre chose. Il y a eu des refroidissements et des réchauffements avant nous.

(8) [Il y] a un cycle planétaire, mais je ne pense pas que l'activité humaines puisse changer le climat. 


\section{Remarques finales}

En général, il semble que les associations thématiques évoquées par l'expression 'changement climatique' sont plus ou moins similaires parmi les Norvégiens et les Français, avec quelques particularités de chaque pays. Plus particulièrement, pour la question de responsabilité globale, les associations sont aussi plus ou moins similaires, indiquant une distinction entre «nous » (dans la plupart des cas réalisé par le pronom 'nous' ou par le nom du pays) et «les autres ». Cependant, selon cette enquête il y a un engagement plus manifeste et plus polémique en Norvège qu'en France. Une explication pourra être la question pétrole versus la question nucléaire, qui posent des défis tout à fait différents dans les deux pays. Il y a également des différences thématiques entre les deux enquêtes. Les Français parlent de perturbations et de catastrophes naturelles, alors que les Norvégiens parlent des conséquences sociales et humanitaires. Ces questions portant sur le contexte social et politique mériteront d'être discutées d'une manière plus approfondie que nous ne l'avons fait ici.

En ce qui concerne les différences identifiées par les analyses linguistiques, ce sont surtout deux observations qui méritent d'être soulignées : la fréquence plus élevée dans les réponses norvégiennes de constructions déontiques et de constructions concessives avec men (mais en français) que dans les réponses françaises. Cette différence indique un plus grand engagement chez les Norvégiens, par le fait de souligner la nécessité d'agir et d'entrer dans une délibération argumentative. Ce résultat est renforcé par la différence en longueur moyenne entre les deux sondages : 10 mots pour les Norvégiens, 5,6 mots pour les Français.

En regardant de plus près les constructions déontiques et concessives chez les Norvégiens, nous observons un flou référentiel et une ambivalence argumentative : on sait qu'il faut agir, mais on n'arrive pas à préciser qui doit agir. On sait que le changement climatique est un phénomène réel, mais on ressent un besoin d'évoquer plusieurs causes naturelles et humaines. Cette incertitude et ambivalence peut illustrer la difficulté de l'opinion publique de prendre acte de ce phénomène complexe, qui a des causes et des effets à la fois naturelles et sociétales. Nous avons vu que les réponses à la question ouverte permettent justement de repérer ces difficultés, alors que les questions fermées d'un questionnaire seraient incapables de les découvrir. Là se montre aussi la pertinence sociétale des deux enquêtes, car la connaissance des préoccupations, incertitudes, doutes et ambivalences chez les répondants peut constituer une ressource importante pour ceux qui ont besoin de s'adresser au public à propos du changement climatique, en vue d'une mobilisation sociale et politique.

\section{Bibliographie}

Birkelund, M., 2009. "Pierre n'est pas français mais danois. Une structure polyphonique à part." Langue française 164: 123-135.

Dahl, T., Fløttum, K., 2014. "A linguistic framework for studying voices and positions in the climate debate." Text \& Talk 34 (4): 401-420.

Fløttum, K. 2016. "Linguistic Analysis Approaches for Assessing Climate Change Communication." In: Climate Science: Oxford Research Encyclopedias. Oxford: Oxford University Press. 
Fløttum, K., 2017. "Willingness of action." In: Fløttum, K. (Ed.) The role of language in the climate change debate. New York/London: Routledge, 113-129.

Fløttum, K., Dahl, T., 2012. "Different Contexts, Different 'Stories'? A Linguistic Comparison of Two Development Reports on Climate Change." Language \& Communication 32 (1): 14-23.

Fløttum, K., Gjerstad, Ø., 2016. "Narratives in climate change discourse." WIREs Climate Change. doi: 10.1002/wcc.429.

Fløttum, K., Gjesdal, A.M., Gjerstad, Ø., Koteyko, N., Salway, A., 2014. "Representations of the future in English language blogs on climate change." Global Environmental Change 29: 213-222.

Gjerstad, Ø., Fløttum, K., 2017. "Negation as a rhetorical tool in climate change discourse." In: M. Roitman (Ed.), The Pragmatics of Negation, Amsterdam: Benjamins, 187-208.

Hulme, M., 2009. Why We Disagree About Climate Change. Cambridge: Cambridge University Press.

Moser, S. C., 2016. "Reflections on Climate Change Communication Research and Practice in the Second Decade of the 21 st Century: What More Is There to Say?" WIREs Climate Change. doi: 10.1002/wcc.403.

Nerlich B, Koteyko N, Brown B., 2010. "Theory and Language of Climate Change Communication." WIREs Climate Change 1: 97-110.

Nølke, H., Fløttum, K., Norén, C., 2004. ScaPoLine. La théorie scandinave de la polyphonie linguistique. Paris : Kimé.

Roberts, M. E., et al., 2014. "Structural topic models for open-ended survey responses." American Journal of Political Science 58: 1064-1082.

Steentjes, K., Pidgeon, N., Poortinga, W., Corner, A., Arnold, A., Böhm, G., Mays, C., Poumadère, M., Ruddat, M., Scheer, D., Sonnberger, M., Tvinnereim, E., 2017. European Perceptions of Climate Change: Topline findings of a survey conducted in four European countries in 2016. Cardiff: Cardiff University.

Stoneman, P., Sturgis, P., Allum, N., 2013. "Exploring public discourses about emerging technologies through statistical clustering of open-ended survey questions." Public Understanding of Science 22: 850-868.

Tvinnereim, E., Fløttum, K., 2015. "Explaining topic prevalence in answers to open-ended survey questions about climate change." Nature Climate Change 5:744-747.

Tvinnereim, E., Fløttum K., Gjerstad, Ø., Johannesson, M.P., Nordø, Å. D., 2017. "Citizens' preferences for tackling climate change. Quantitative and qualitative analyses of their freely formulated solutions." Global Environmental Change 46: 34-41. 